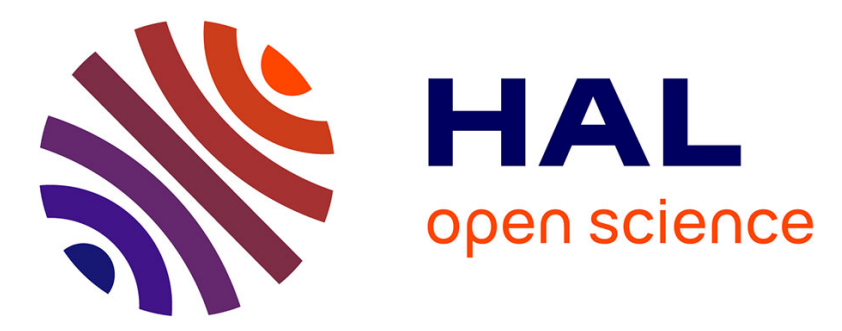

\title{
Image analysis characterization of multi-walled carbon nanotubes
}

\author{
C. Gommes, S. Blacher, Karine Masenelli-Varlot, Ch. Bossuot, E. Mcrae, A. \\ Fonseca, J. B Nagy, Jp Pirard
}

\section{> To cite this version:}

C. Gommes, S. Blacher, Karine Masenelli-Varlot, Ch. Bossuot, E. Mcrae, et al.. Image analysis characterization of multi-walled carbon nanotubes. Carbon, 2003, 41 (13), pp.2561-2572. 10.1016/S00086223(03)00375-0 . hal-00475119

\section{HAL Id: hal-00475119 \\ https://hal.science/hal-00475119}

Submitted on 13 Jun 2019

HAL is a multi-disciplinary open access archive for the deposit and dissemination of scientific research documents, whether they are published or not. The documents may come from teaching and research institutions in France or abroad, or from public or private research centers.
L'archive ouverte pluridisciplinaire HAL, est destinée au dépôt et à la diffusion de documents scientifiques de niveau recherche, publiés ou non, émanant des établissements d'enseignement et de recherche français ou étrangers, des laboratoires publics ou privés. 


\title{
Image analysis characterization of multi-walled carbon nanotubes
}

\author{
C. Gommes ${ }^{\mathrm{a}, *}$, S. Blacher ${ }^{\mathrm{a}}$, K. Masenelli-Varlot ${ }^{\mathrm{b}}$, Ch. Bossuot ${ }^{\mathrm{a}}$, E. McRae $^{\mathrm{c}}$, \\ A. Fonseca ${ }^{\text {, }}$ J.-B. Nagy ${ }^{\mathrm{d}}$, J.-P. Pirard ${ }^{\mathrm{a}}$ \\ ${ }^{a}$ Laboratoire de Génie Chimique, Université de Liège, Bâtiment B6a, B-4000 Liège, Belgium \\ ${ }^{\mathrm{b}}$ Groupe d'Études de Métallurgie Physique et de Physique des Matériaux, UMR CNRS 5510, INSA de Lyon, Bâtiment B. Pascal, \\ 7 Avenue J. Capelle, F-69621 Villeurbanne Cedex, France \\ ${ }^{\mathrm{c}}$ Laboratoire de Chimie du Solide Minéral, UMR CNRS 7555, Université Henri Poincaré Nancy I, B.P. 139, \\ F-54506 Vandoeuvre les Nancy Cedex, France \\ ${ }^{\mathrm{d}}$ Laboratoire de Résonance Magnétique Nucléaire, FUNDP, 61 Rue de Bruxelles, B-5000 Namur, Belgium
}

Received 7 January 2003; accepted 14 July 2003

\begin{abstract}
An original image analysis method is presented to characterize multi-walled carbon nanotubes from transmission electron microscopy images. The analysis is performed in three steps: (i) image preprocessing in order to isolate the nanotubes from the background, (ii) image segmentation, aiming at keeping only the measurable sections of nanotubes, and finally (iii) tube characteristics measurement. The measurement is based on a Lambert-like electron absorption law and is performed on the original gray level image itself. Two geometrical and one physical characteristics are determined for each tube, namely, its outer and inner radius and a linear electron absorption coefficient. The method is illustrated by comparing a pristine and an annealed carbon nanotube samples. The compaction of the tube walls during annealing is shown to result from a lowering of the external radius while the inner radius is left unchanged.
\end{abstract}

(C) 2003 Elsevier Ltd. All rights reserved.

Keywords: A. Carbon nanotubes; C. Transmission electron microscopy (TEM), Image analysis

\section{Introduction}

Since their discovery by Ijima in 1991 [1], carbon nanotubes have been found to possess many interesting properties and potential applications [2]. A considerable amount of work has thus been devoted to improve their production using different physical and chemical methods (arc discharge, catalytic decomposition of hydrocarbons, laser ablation, etc.) [3]. A growing number of research projects is under way to upscale the production capability in order to reach an economically viable level. As the upscaling of a production facility is known to be a very delicate task, the need for reliable characterization tools is becoming more and more essential.

Transmission electron microscopy (TEM) is a common-

\footnotetext{
*Corresponding author. Tel.: +32-4-366-2951; fax: +32-4366-3545.

E-mail address: cedric.gommes@ulg.ac.be (C. Gommes).
}

ly used technique to characterize nanotube samples. Some general properties of the observed sample such as the mean inner and outer radii, the mean tube length are inferred from the micrographs. The information obtained in that way can be affected by two types of errors. Firstly, the experimenter is usually prone to restrict the analysis to a given part of the image that is judged the most representative, while rejecting the rest of the image. The criteria used for choosing the zone to be analyzed are generally based on a subjective visual analysis, leading thus to a bias error in the measurement. Secondly, any quantitative measurement so-performed on a statistically relevant number of images is very cumbersome to obtain. The total number of analyzed images is usually small. The sampling might thus be insufficient to reach a reasonable confidence interval on any measurement.

Quantitative texture analysis of multi-walled nanotubes requires knowledge of both inner and outer radius distributions. In this paper a computer assisted image analysis 
methodology is proposed to determine these parameters based on a large number of TEM micrographs.

The preprocessing algorithms used are based on well known tools of signal processing and mathematical morphology ([4,5] and references therein). Their strict definitions are beyond the scope of this paper, and we will focus on their physical meanings.

The measurements of the inner and outer radii are performed on the gray level images themselves and are based on a Lambert-like electron absorption law. This physical approach further allows the nanotubes to be characterized by their linear electron absorption coefficient.

Sensitivity of the method is tested by comparing a pristine and an annealed carbon nanotube sample.

\section{Material and methods}

The multi-walled nanotubes used for illustrating this paper were provided by the CRMD Laboratory of Orléans (France). They were prepared by catalytic decomposition of acetylene diluted in nitrogen at $600{ }^{\circ} \mathrm{C}$ on $\mathrm{Co}_{x} \mathrm{Mg}_{1-x} \mathrm{O}$, as described elsewhere [6]. The nanotubes were further annealed at $2500{ }^{\circ} \mathrm{C}$ under an argon flux in order to remove metallic impurities and to reduce the number of defects in the nanotube walls.

The multi-walled nanotubes were ultrasonically dispersed in ethanol. A drop of the solution was then deposited on a copper 400-mesh microscopy grid covered with a carbon film.

The TEM micrographs were obtained using a Philips CM20 microscope, equipped with a LaB6 filament. The operating voltage was set to $200 \mathrm{kV}$ and the magnification to 200,000 . The images were collected in bright field mode. An objective aperture $(50 \mu \mathrm{m})$ was used to enhance the contrast of the images. These experimental conditions allowed a good discrimination between the nanotubes and the background. The TEM micrographs were digitized on a $850 \times 650$ matrix with 256 gray levels, leading to a resolution of $0.423 \mathrm{~nm}$ per pixel. Fifteen images were acquired and used for image analysis.

The image treatment and subsequent statistical analysis were performed using the Matlab software and its image processing toolbox.

\section{Results}

\subsection{Image analysis}

Fig. 1 shows a typical transmission electron micrograph of a multi-walled nanotubes sample. As can be observed, two main difficulties arise which prevent performing standard measurements. On one hand, the contrast between nanotubes and the background is made uneven by the

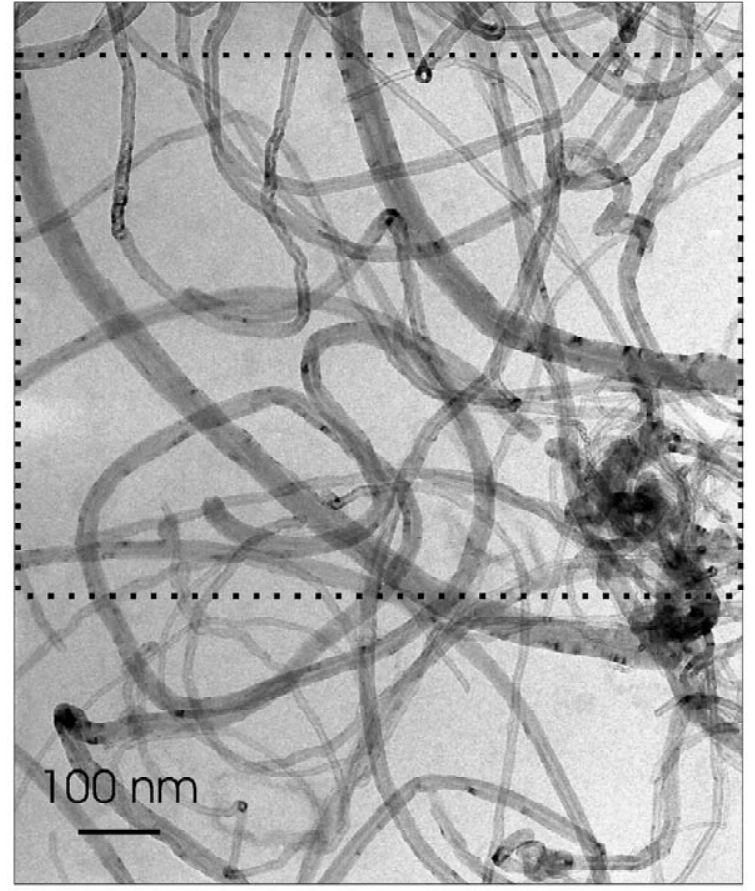

Fig. 1. Typical transmission electron microscopy micrograph of multi-walled carbon nanotubes. The inset is the part of the image that is used for illustrating the proposed analysis.

irregular illumination of the sample by the electron beam. On the other hand there are regions in which nanotubes are so entangled so as to make any local measurement impossible. Image analysis will thus be divided into the following three steps: (i) image preprocessing in order to isolate the nanotube set from the background, (ii) image segmentation to determine which parts of the nanotubes are measurable, (iii) measurement of the selected sections of nanotubes. This last step is performed on the original gray level images themselves.

\subsubsection{Preprocessing}

The main preprocessing steps are illustrated in Fig. 2. Fig. 2a presents the original image. As a first step, a noise suppressing median filter is applied to the image (Fig. 2b). In order to allow every part of the image to be treated on the same footing, one first has to remove the effect of the uneven illumination of the sample. Traditionally this could be performed with a high-pass linear filtering, using for instance a Fourier transform. However, this would result in a loss of the information contained in the intensity of the image. As discussed later, the analysis proposed in this work is mainly based on the variation of the image intensity across a nanotube. Therefore the background correction cannot consist in a mere subtraction but must take into account the physical phenomena of image formation (see Section 3.1.3). Consequently, the following 

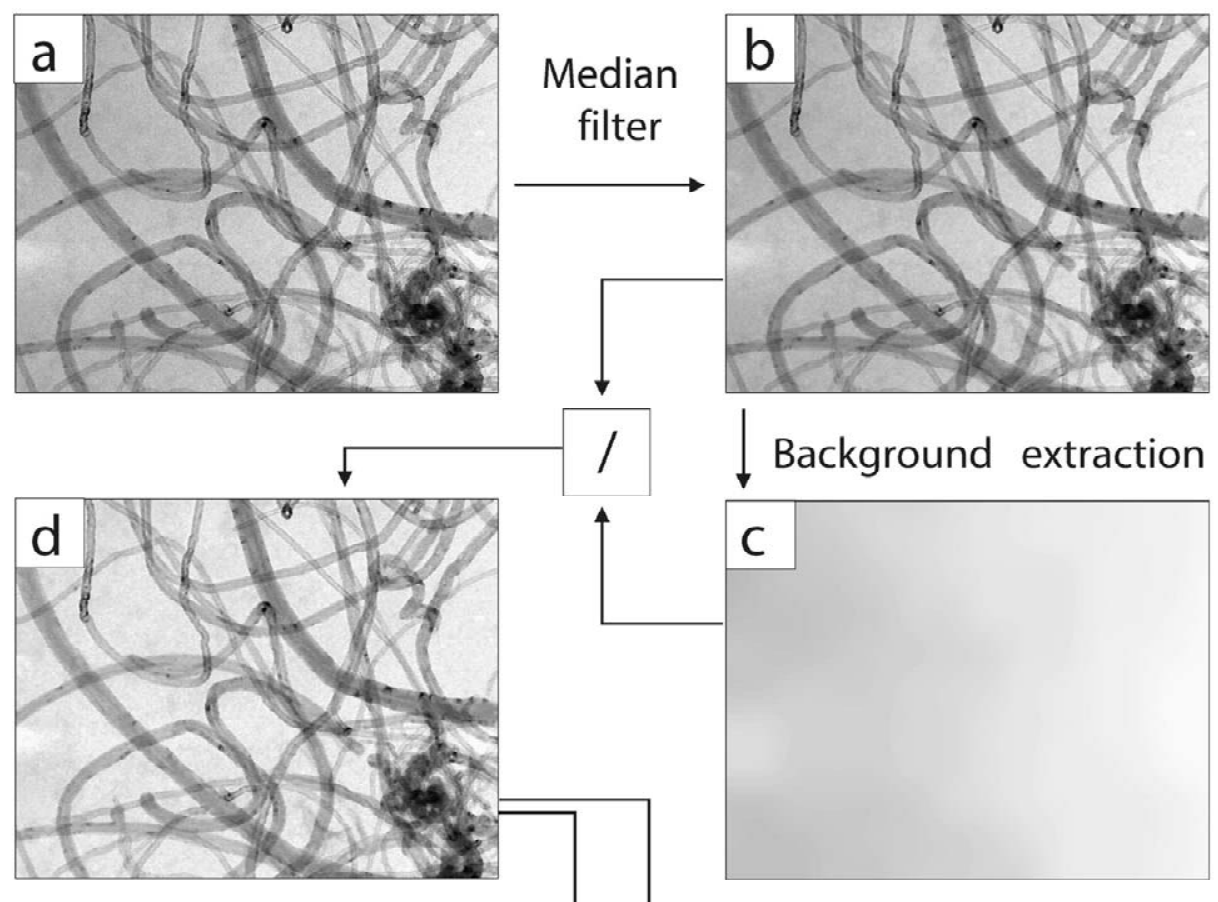

Loose threshold
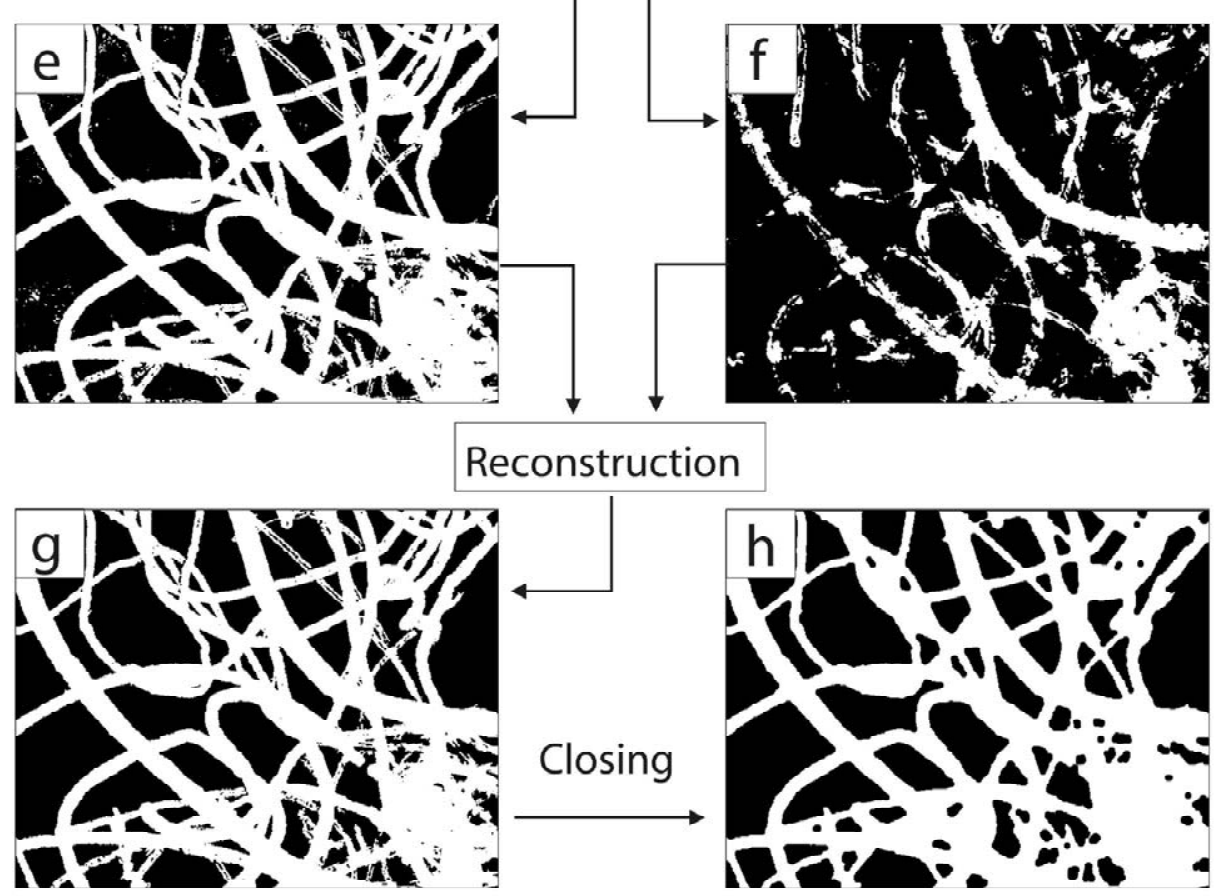

Fig. 2. Preprocessing steps: (a) original image, (b) resulting image after median filtering, (c) background intensity in the original image, (d) illumination corrected image, (e) and (f) binary images resulting from a low and high thresholds, (g) binary image containing the objects of (e) also present in (f), (h) final binary image. 
method is used: first the maximum intensity of each of the $50 \times 50$ pixel blocks of the images is calculated. This coarse estimate is then expanded in size using a bicubic interpolation, so that it has the same size as the original image (Fig. 2c). The illumination of the sample is made even by dividing the original image by the background illumination. While the illumination in Fig. 2a is more intense in the right part of the image, this effect has disappeared in Fig. 2d.

The image is then binarized according to the double threshold technique [4,5]. A loose and a severe threshold are applied to the same image, leading to two black and white images $I_{\mathrm{L}}$ (Fig. 2e) and $I_{\mathrm{S}}$ (Fig. 2f). The binary image (Fig. 2g) is then obtained by keeping the connected white objects of $I_{\mathrm{L}}$ that are also present in $I_{\mathrm{S}}$. The choice of these two thresholds is based on Otsu's method [7], which allows determining the threshold that minimizes the intraclass variance of the black and white pixels.

Because of the noise, the white objects remaining in the images after this step usually contain small black holes. These can be removed by applying a filter called closing [4] that smoothes the white regions of the image. The final binary image, BW, is shown in Fig. $2 \mathrm{~h}$.

\subsubsection{Segmentation}

The binary image BW (Fig. 3a) has all its white pixels on the measurable sections of the nanotubes and also on the crossings of the entangled nanotubes. These latter parts of the image have to be removed prior to any reliable measurement.

The method chosen to separate the different portions of the nanotubes is inspired by the algorithm developed by Soille [4] for separating fibers. The algorithm proposed in the present study divides into several steps. Step i: nanotubes of the binary image (Fig. 3a) are reduced to their middle line by applying a succession of thinning operations called skeletonization. Because of the binarized nanotube roughness, the skeleton contains many branches that do not lie in the middle of the nanotubes but join the nanotube center to the nanotube boundary. These spurious small skeletal branches (not shown) are eliminated using a parametric pruning. Fig. $3 \mathrm{~b}$ shows the final pruned skeleton. Step ii: the distance transform of the binary image is computed (Fig. 3c). By applying a distance transform, every white pixel of an image is assigned a value equal to the distance to its closest black pixel. For a region corresponding to a section of a nanotube, the distance at the pixels of the skeleton is equal to the tube's radius. For regions corresponding to the crossing of several tubes, the distance at the pixels of the skeleton are a rough estimate of the size of the region to be removed. Step iii: a mask is created by replacing every crossing point of the skeleton by a disk whose radius is larger than the value of the distance at that point (Fig. 3d). Step iv: nanotube separation and removal of the entanglement zones is performed by subtracting this mask from the binary image
(Fig. 3e). The size of the disks used in the mask creation is a critical parameter. If the disks are chosen too small, then the sections of the nanotubes would not disconnect when the mask is subtracted. On the contrary, if it is too large, some measurable sections of nanotubes would be eliminated. In our case, the choice of disks 50\% larger than the distance at the corresponding crossing points resulted in a suitable compromise. Step v: elimination of the spurious objects still remaining in the segmented images. These are objects touching the borders of the image and artifacts. The former are removed using a so-called border-kill operation (already applied in Fig. 3e). The latter cannot be eliminated using a traditional size criterion for this would introduce a bias in the measurement. Indeed, this would remove indiscriminately the artifacts and the smaller nanotubes. Therefore, a criterion based on the length of the branch of the skeleton (Fig. 3b) corresponding to a given object was used. The image resulting from the intersection of the segmented image (Fig. 3e) and the skeleton (Fig. 3b) contains information on the lengths of the nanotubes sections independently of their area (Fig. 3f). Therefore, size filtering can be applied to this image without affecting the diameter distribution of the nanotubes. The resulting image (Fig. 3g) contains only those branches of Fig. 3f which contain at least 10 pixels. The corresponding sections of nanotubes are represented in Fig. 3h, which is the final result of the preprocessing.

Fig. 4a superimposes the original gray-level image and the boundaries of the parts of it selected for analysis. These retained zones almost all correspond to neat nanotube sections which are worth being measured. Furthermore, there are very few nanotubes in the image that do not contain at least one selected measurable zone.

\subsubsection{Quantification}

Measurable sections of nanotubes appear as cylinders with a given gray level distribution, as can be seen from Fig. 4b, c and d. A local maximum intensity can be seen along the tube's axis, which corresponds to its inner hollow cavity. Going away from the axis, the intensity decreases and reaches a minimum in the wall, and eventually increases again as one leaves the tube. The determination of the intensity profile across a nanotube allows thus the inner and outer radii to be estimated.

The principle of intensity profile measurement is the following, (i) the skeleton branch, which lies in the middle of its corresponding tube, is traced (Fig. 5a), (ii) the distance transform from the skeleton is determined (Fig. $5 b$ ), and (iii) the intensity profile is obtained by averaging the intensity of all pixels of the gray level image located at a given distance from the skeleton (Fig. 5d).

In practice, it is necessary to isolate each tube for determining its intensity profile. This could be performed by using the corresponding part of the binary image as a mask. However, as the use of the intensity profile aims at 

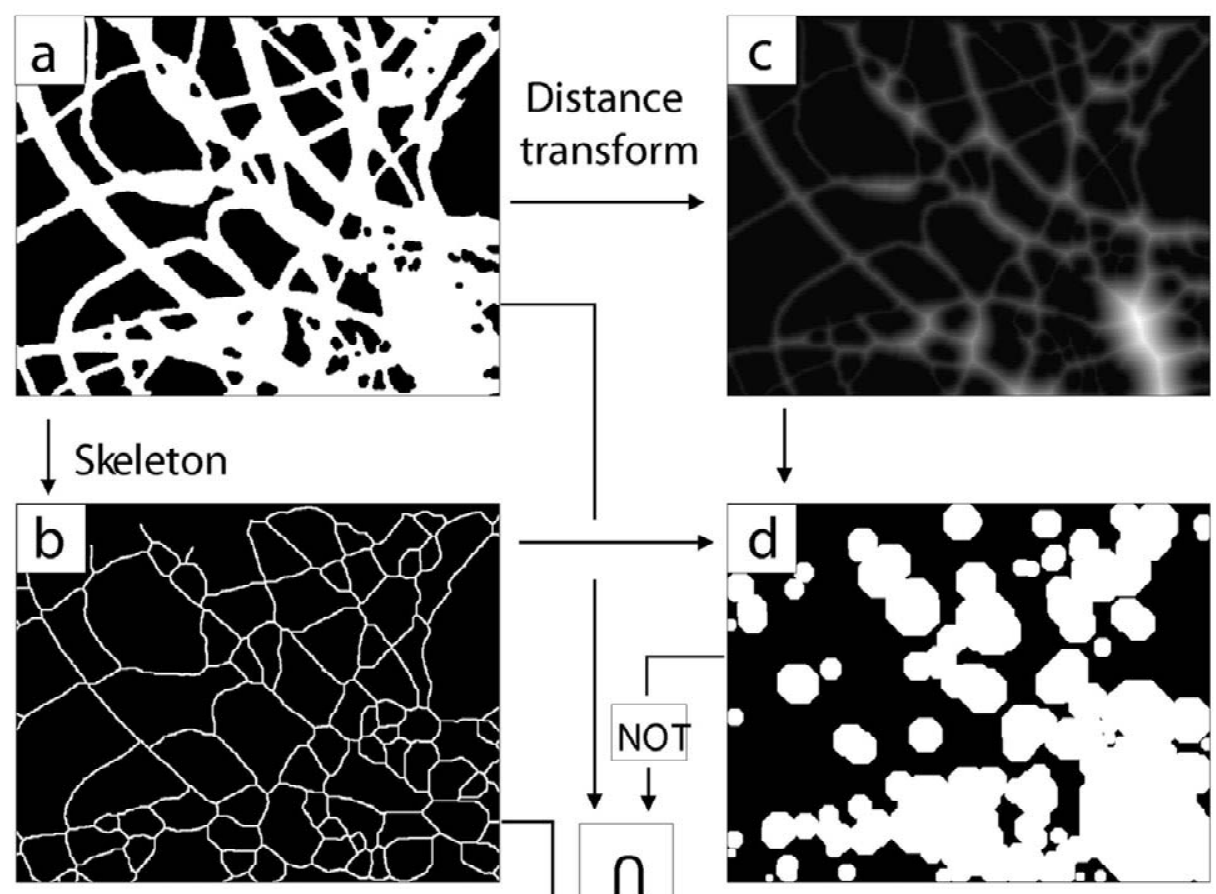

Distance transform
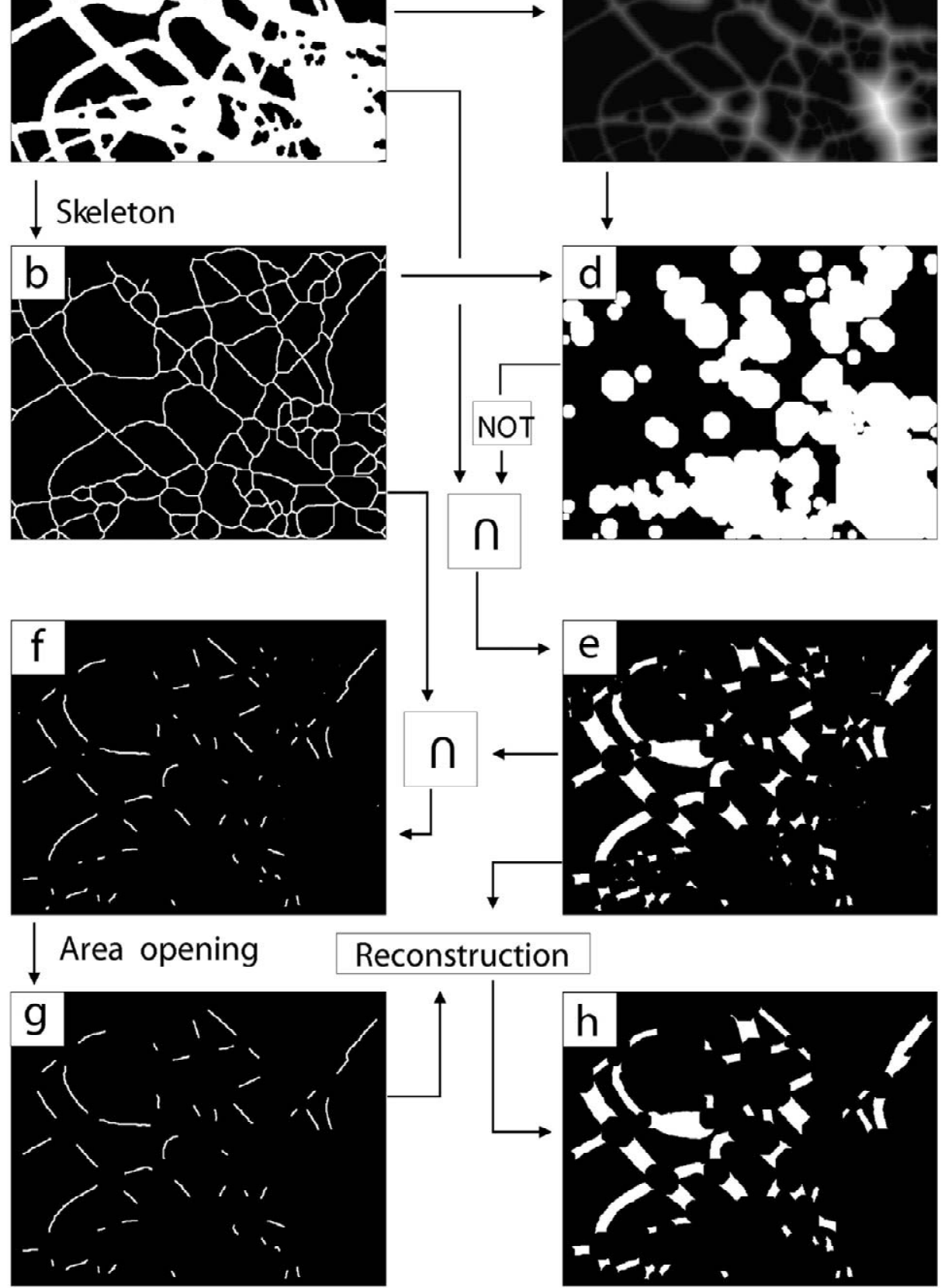

Fig. 3. Segmentation steps: (a) binary image, (b) middle lines obtained by skeletonization and pruning, (c) distance image, (d) dilated crossing points of the skeleton, (e) segmented image resulting from the subtraction of image (a) and (d), (f) intersection of images (b) and (e), (g) area filtering of image (f), (h) final segmented image obtained by keeping the objects of (e) that are present in (g). 


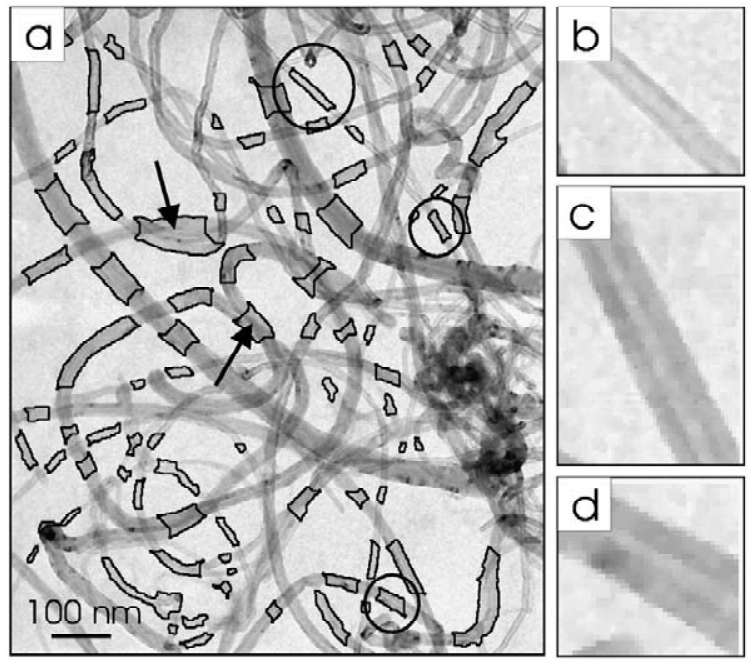

Fig. 4. Final result of the preprocessing and segmentation procedures: (a) superimposition of the selected sections of nanotubes and of the original image, (b), (c) and (d) are magnification of some selected tubes (indicated by a circle) showing the characteristic gray level distribution. Some parasitic objects are indicated by an arrow. comparing the gray levels associated with a nanotube to the gray levels associated with the background, this mask has to be chosen large enough as to contain some background pixels. Therefore, the image of the binary nanotubes (Fig. 3h) is dilated and each piece is used to select the corresponding part of the gray level image. As can be seen from Fig. 5c this allows to take only the neighborhood of a given nanotube into account for computing its intensity profile.

Fig. 5d shows a typical intensity profile. At large distances from the skeleton, the intensity of the image is the same as the background intensity, $I_{0}$. As the distance to the skeleton becomes smaller, the intensity decreases, and reaches a minimum, corresponding to the nanotube wall. Very close to the tube center, in the region corresponding to the hollow cavity, the intensity eventually increases again.

In most cases no clear cut transition exists on the intensity profile, neither at the outer nor at the inner limit of the wall. It is thus useful to model the image and electron absorption by the tube material. This can be done using Lambert's law [8]. Namely, if $I_{0}$ is the intensity of the incident electron beam, the intensity $I$ of the same beam after having crossed a thickness $t$ of material obeys:
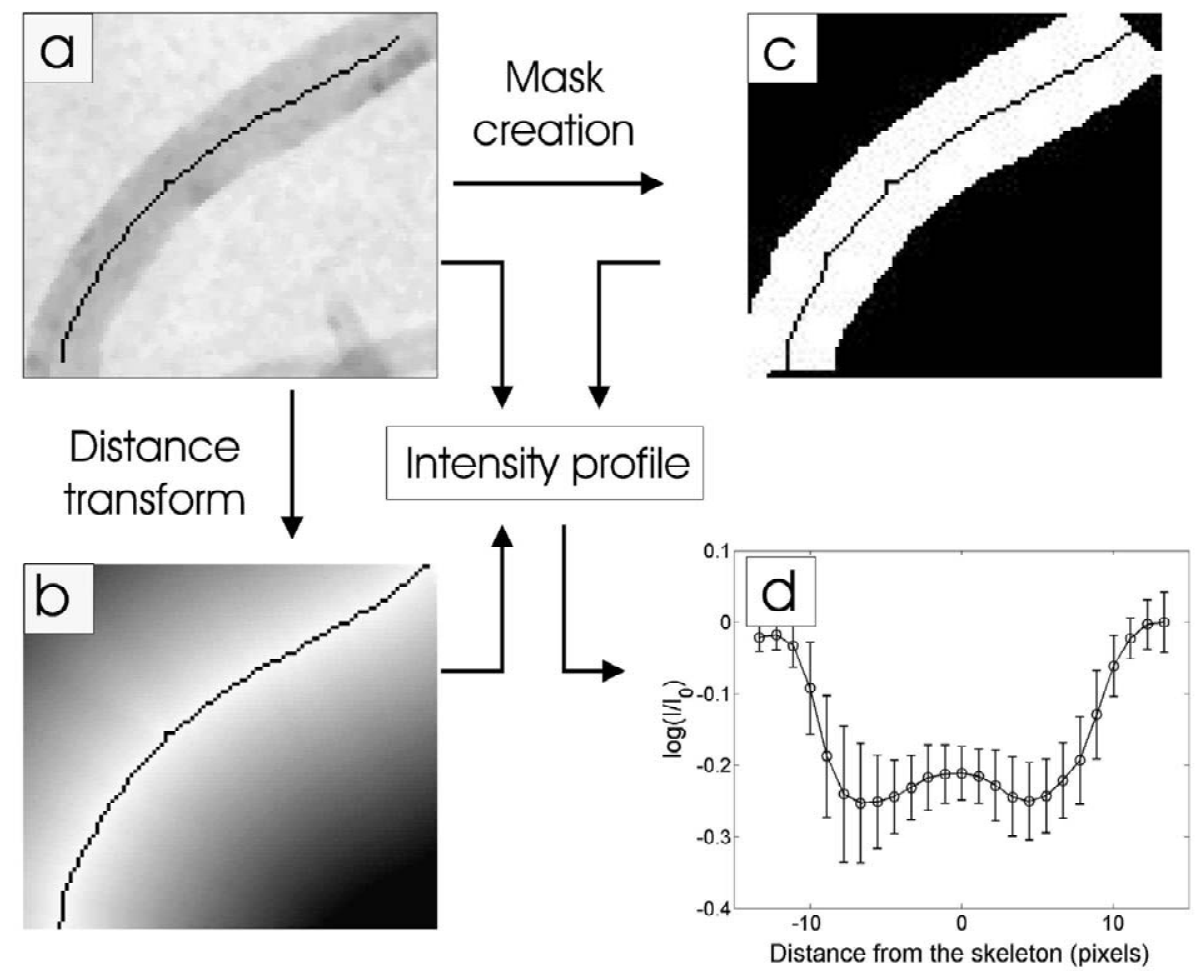

Fig. 5. Principle of intensity profile determination: (a) gray level image of a selected nanotube with its skeletal branch, (b) distance transform from the skeleton branch, (c) mask creation. The intensity profile (d) is measured by averaging the intensity of every pixel of the mask located at a given distance from the skeleton. 
$\log \left(\frac{I}{I_{0}}\right)=-k t$

where $k$ is a linear absorption coefficient. This coefficient is a characteristic of the wall's material.

In the case of a perfect hollow cylinder, with outer radius $R_{\mathrm{O}}$ and inner radius $R_{\mathrm{I}}$ (Fig. 6), it is an easy matter to compute the thickness of material, $t$, crossed by the electrons as a function of the distance, $d$, from the cylinder's center. Introducing this relation in Lambert law the following relation is obtained:

$$
\begin{aligned}
& -\log \left(\frac{I}{I_{0}}\right) \\
& = \begin{cases}0 & \text { if } d>R_{\mathrm{O}} \\
2 k R_{\mathrm{O}} R_{\mathrm{O}} \sqrt{1-\left(d / R_{\mathrm{O}}\right)^{2}} & \text { if } R_{\mathrm{O}} \geq d \geq R_{\mathrm{I}} \\
2 k\left[R_{\mathrm{O}} \sqrt{1-\left(d / R_{\mathrm{O}}\right)^{2}}-R_{\mathrm{I}} \sqrt{1-\left(d / R_{\mathrm{I}}\right)^{2}}\right] & \text { if } R_{\mathrm{I}}>d\end{cases}
\end{aligned}
$$

This function can be fitted to the measured profile using a non linear least squares minimization technique [10], leading to numerical values of $R_{\mathrm{O}}, R_{\mathrm{I}}$ and $k$. Every non linear minimization procedure requires a first estimated value of every parameter to be optimized. The outer radius is estimated at first as half the ratio of a section area to the length of its skeletal branch. The internal radius is estimated as the distance from the skeleton leading to the lowest intensity, $I_{\min }$. A first value of the linear absorption coefficient is chosen as:

$k \approx-\frac{1}{2} \cdot \frac{\log \left(I_{\min } / I_{\mathrm{O}}\right)}{\sqrt{R_{\mathrm{O}}^{2}-R_{\mathrm{I}}^{2}}}$

which is obtained by isolating $k$ in Eq. (2) with $d=R_{\mathrm{I}}$ and $I=I_{\min }$.

Fig. 7a shows an example of successfully fitted intensity profile. Despite its simplicity, the Lambert model seems to catch the essential of the physical phenomena implied in the image formation.

Some parasitic objects passed through the selection criteria applied to the binary image, such as the ones indicated by arrows in Fig. 4a. These objects usually correspond to the small angle crossing of two nanotubes. The intensity profiles of such objects usually do not exhibit a local maximum at $d=0$, as visible from Fig. 7b, and the least squares fit leads to $R_{\mathrm{I}}=0$. These objects should be rejected. It would also make no sense to take into account measurements whose order of magnitude is lower than the image resolution. Every object leading to an inner radius lower than one pixel was therefore not taken into account in the statistical analysis.

\subsection{Influence of annealing on the estimated parameters}

This image analysis method was applied in order to determine the effect of annealing on a sample of nanotubes under an argon flux. This annealing procedure is known to remove any metal impurities and to improve the overall crystallinity of the nanotubes [9].

One representative image of the sample before and after annealing is presented in Fig. 8a and b, respectively. At first sight, no significant difference can be seen between these two images. However a closer look at some details of the images reveals that the pristine nanotubes have a rougher surface, and that the inner and outer radii of a given nanotube is more variable than in the annealed sample.

The methodology presented in the preceding paragraph was applied to two series of 15 images taken from each sample. The parts of each image suitable for measurement where isolated and their corresponding intensity profiles were determined. By fitting the Lambert model to the intensity profiles, the outer radius, the inner radius and the linear absorption coefficient of every selected tube portion was estimated.

In estimating the distribution of these variables, each

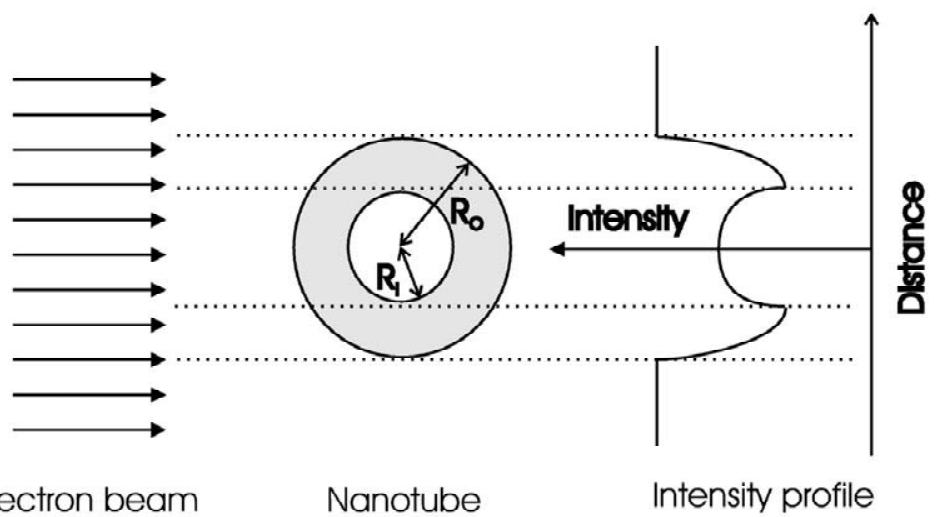

Fig. 6. Intensity profile modeling assuming a perfectly cylindrical geometry and using a Lambert law for the electron absorption by the wall. 

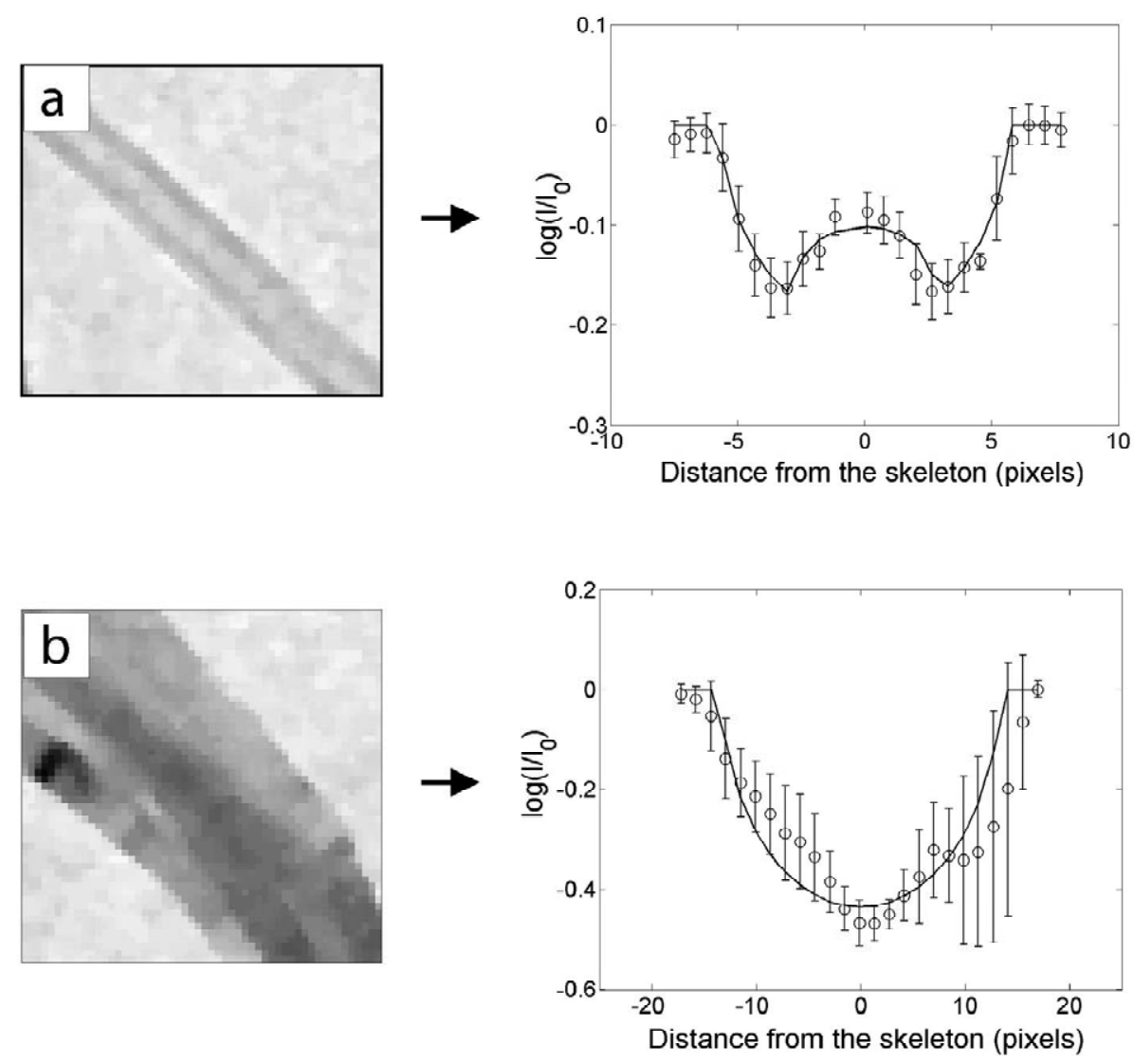

Fig. 7. Examples of selected objects and their fitted intensity profiles. Case (a) is a nanotube, while case (b) is a parasitic object resulting from the low-angle crossing of two nanotubes.

section was weighed by the length of its skeleton. Doing so ensures that the distributions are not modified when a given nanotube is sectioned into several small portions by the preprocessing step. The distributions obtained thus correspond to the distributions of the tube lengths as a function of the estimated characteristic parameters.

The estimated distributions are shown in Fig. 9, in which black bars correspond to pristine sample and white bars to same sample after annealing. A KolmogorovSmirnov (K-S) test [10] was performed for each estimated tube characteristic in order to test whether its distribution was affected by annealing. The significance level $Q_{\mathrm{KS}}$ of the $\mathrm{K}-\mathrm{S}$ test for each tube characteristic is shown in Table 1. This quantity has to be interpreted as the probability that the difference between the two estimated distributions would be larger than observed by accident if the distributions were identical. With a significance level of $10 \%$ it is shown that, the distributions of the outer radius, of the wall thickness, and of the Lambert absorption coefficient are significantly modified by annealing. The variations of the inner radius are not statistically significant.

\section{Discussion}

\subsection{General methodology}

An original three-step image analysis software has been developed to characterize multi-walled carbon nanotubes from TEM images. The first two steps are preprocessing ones. The first step aims at separating nanotubes from the background. The second step isolates every measurable tube section. The third and last step is the tube characteristics measurement itself performed on the so selected parts of the gray level images.

The measurement is based on the image's intensity profile across a nanotube. The intensity profile is modeled assuming the tube geometry is a perfect hollow cylinder, and that the electron absorption by the tube material obeys Lambert's law. Fitting the model intensity on every determined profile allows the outer and inner radii of the tubes to be estimated as well as their linear electron absorption coefficient.

The measurement step is free of any user defined 

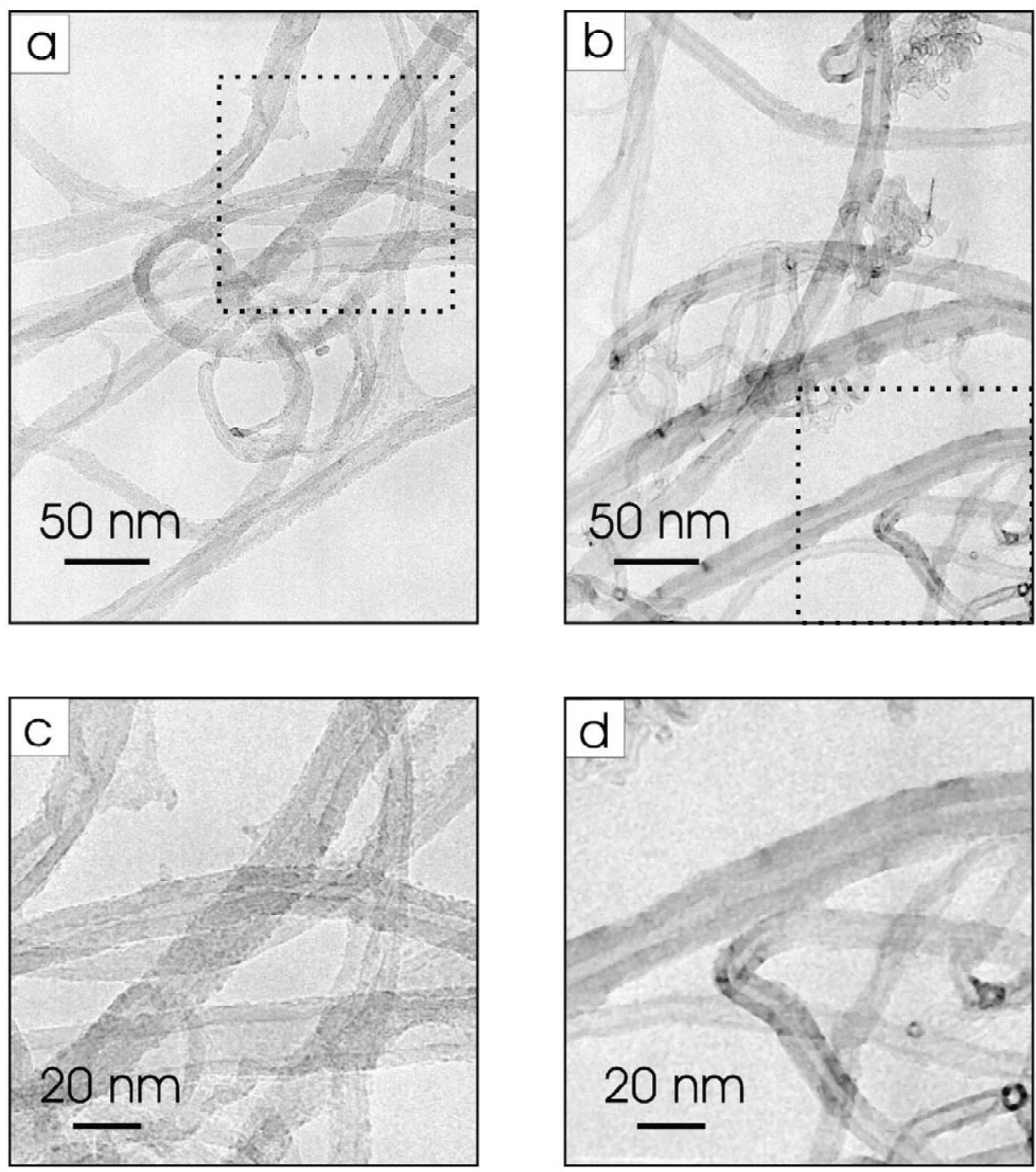

Fig. 8. Representative TEM micrographs of the pristine (a) and annealed (b) nanotube sample. (c) and (d) are enlargements of the regions delimited by the dotted line of images (a) and (b), respectively.

parameter, but some of the transformations used during the preprocessing are parametric. The choice of every parameter is based on a compromise between removing as many unsuitable parts of the image and keeping as many suitable parts as possible. This choice cannot be independent of the images. Important image characteristics include their noisiness and the size of the tubes. The user should therefore optimize the preprocessing parameters on a small number of representative images. Once the optimization step is achieved, the parameters are left unchanged and a large series of images can be treated.

It should be emphasized that the measurements are performed on the unprocessed images, whose information is sure not to have been damaged by the preprocessing.
Therefore, should the parametric steps of the analysis be badly optimized, this would lead to the presence of many parasitic objects or of too few nanotubes in the images, but this would not modify the values of the estimated characteristics of a given nanotube.

Some of the drawbacks of visual inspection of the micrographs have been removed with the proposed methodology. The criteria used to choose the parts of the images to be analyzed are made totally objective. These criteria might be criticized but insofar as the same criteria are used to analyze several series, these can be reasonably compared. In that respect, the present measurement procedure is less biased than visual inspection. Furthermore, once the preprocessing parameters have been chosen, the 

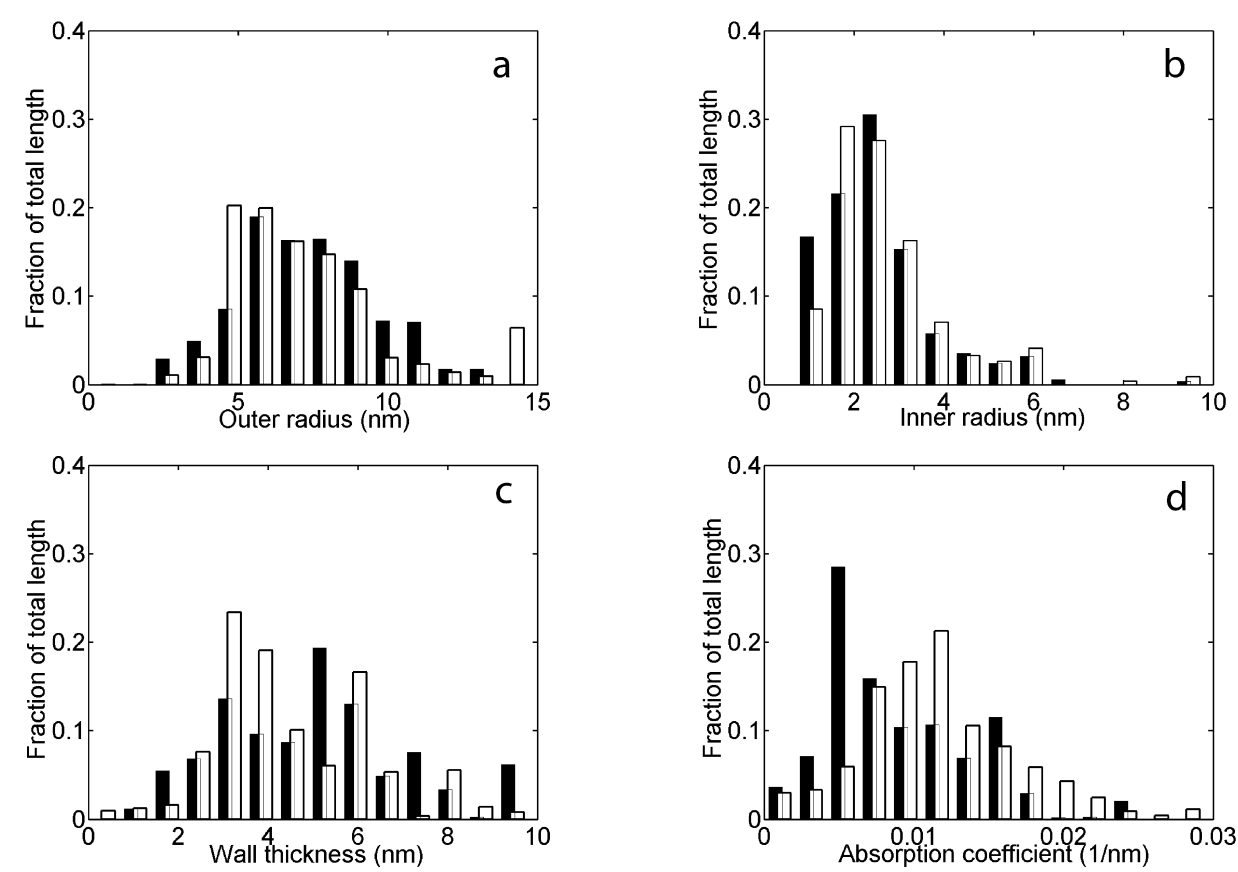

Fig. 9. Statistical distributions of the nanotubes length as a function of outer radii (a), inner radii (b), wall thickness (c) and linear electron absorption coefficient (d) of the pristine (black bars) and annealed nanotube sample (white bars), determined by image analysis.

treatment is entirely automatic and a statistically relevant number of images can be used.

In order to test the reliability of the automatic image analysis, the outer and inner radius distribution of the same samples were determined manually (Fig. 10 and Table 1). When performing the manual quantification, all nanotubes visible in the images where counted once. This resulted in 151 visible nanotubes in the 15 images of the pristine sample, and 162 nanotubes in the annealed sample. Comparatively, the automated procedure resulted in 102 selected sections for the pristine sample and 171 sections for the annealed sample. Since each nanotube can be segmented into several pieces, the automated method should lead, as in the case of the annealed sample, to more measured sections than the actual number of nanotubes. However, when the entanglement is too large, some nanotubes do not lead to any automatically measurable section, as in the case of the pristine sample. Therefore, in order to avoid the bias that would be introduced by this effect, the proposed statistics is based on the length of the measured sections rather than on their number.

From the data reported in Table 1, it can be seen that the radii measured manually are slightly lower than the same radii estimated by the image analysis. This can be attributed to the difference of perception between the human eye and the fitting procedure of the smooth transition between

Table 1

Mean value and standard deviation of the estimated characteristics of the pristine and annealed samples

\begin{tabular}{|c|c|c|c|c|c|}
\hline & $\begin{array}{l}R_{\mathrm{O}} \\
(\mathrm{nm})\end{array}$ & $\begin{array}{l}R_{\mathrm{I}} \\
(\mathrm{nm})\end{array}$ & $\begin{array}{l}T \\
(\mathrm{~nm})\end{array}$ & $\begin{array}{l}k \\
(1000 / \mathrm{nm})\end{array}$ & $\begin{array}{l}k\left(R_{\mathrm{O}}^{2}-R_{\mathrm{I}}^{2}\right) \\
(\mathrm{nm})\end{array}$ \\
\hline Pristine & $\begin{array}{l}7.2 \pm 2.4 \\
(6.2 \pm 2.1)\end{array}$ & $\begin{array}{l}2.4 \pm 1.0 \\
(2.4 \pm 1.0)\end{array}$ & $\begin{array}{l}4.8 \pm 2.1 \\
(3.8 \pm 1.5)\end{array}$ & $9.2 \pm 4.6$ & $0.39 \pm 0.24$ \\
\hline Annealed & $\begin{array}{l}6.7 \pm 2.6 \\
(6.0 \pm 2.2)\end{array}$ & $\begin{array}{l}2.5 \pm 1.1 \\
(2.3 \pm 0.9)\end{array}$ & $\begin{array}{l}4.2 \pm 2.0 \\
(3.7 \pm 1.6)\end{array}$ & $13.6 \pm 9.2$ & $0.46 \pm 0.35$ \\
\hline$Q_{\mathrm{Ks}}$ & 0.00223 & 0.2779 & $6.29 \times 10^{-4}$ & $1.60 \times 10^{-6}$ & 0.5999 \\
\hline Variation $(\%)$ & $-6.9^{\mathrm{a}}$ & +4.2 & $-12.5^{\mathrm{a}}$ & $+47.8^{\mathrm{a}}$ & +17.9 \\
\hline
\end{tabular}

$R_{\mathrm{O}}$ : outer radius; $R_{\mathrm{I}}$ : inner radius, $T$ : wall thickness; $k$ : linear electron absorption coefficient. Quantities in parentheses correspond to the distributions obtained manually.

${ }^{\text {a }}$ Statistically significant variations according to a Kolmogorov-Smirnov test with $Q_{\mathrm{KS}}<0.1$. 

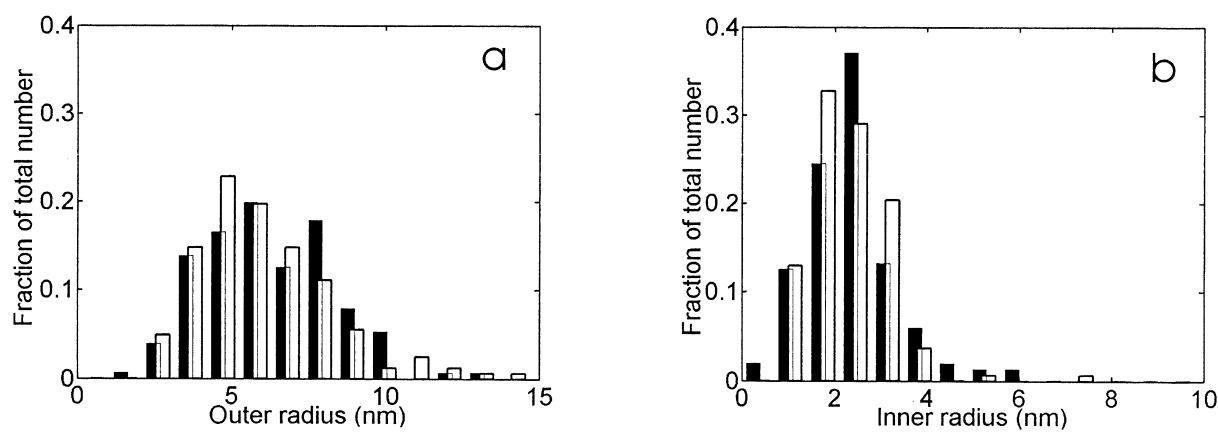

Fig. 10. Statistical distributions of the nanotubes number as a function of outer radii (a), inner radii (b), determined manually.

the nanotube and the background intensities. Moreover, the observed differences are much lower than the standard deviations of the corresponding measurements.

An important issue concerns the number of TEM images to be used for a measurement to be representative. In order to answer this question, it must be reminded that a typical characterization results from the following steps: (i) from a sample of approximately $1 \mathrm{~g}$ of nanotubes, a few $\mathrm{mg}$ are taken and diluted in ethanol, (ii) from the few $\mathrm{ml}$ of ethanol, a drop is taken and deposited on the grid, (iii) from the many meshes of the grid, only a few are visited by the experimenter. Therefore the procedure to be adopted depends on the scale at which the sample is heterogeneous. If heterogeneity arises at a fine scale, then the question of the number of images is relevant. On the contrary, if heterogeneity arises on a large scale, the question of the number of grids should be addressed.

In our case, we supposed that the dispersion in ethanol was sufficient to homogenize the sample, and therefore to make the droplet representative. Then, the analysis of more than the considered number of images would not necessarily make the measurement more precise. In any case, every conclusion that could be drawn from the observation of the micrographs depends finally on the homogeneity of the sampling. Therefore no general rule exists independently of the precise nature of the studied material.

\subsection{Tube characteristics measurement}

The characteristics of the nanotubes determined by the proposed method are of two types. The outer and inner radii are geometric parameters which are classically within the reach of image analysis techniques. The Lambert linear absorption coefficient is a physical characteristic of the tube walls. This latter parameters renders quantitative the observation that for a given thickness a tube may appear more or less dark. It should be stressed that this observation can by no means be made quantitative in the case of a simple visual inspection of the micrographs.

Intuitively, one is prone to conclude that if the electron loss in a given thickness of matter is larger, then the material should be denser. However, in well organized material such as nanotubes, a diffraction mechanism is also responsible for the contrast of the images. No clear cut conclusion can therefore be drawn concerning the exact interpretation of the measured linear absorption coefficient.

The very use of a single absorption coefficient means that the material is supposed to be isotropic. A more realistic model taking absorption anisotropy into account, could be used to fit the determined intensity profiles. For the sake of simplicity a simpler model was used in the present study. The estimated absorption coefficient is to be considered as a parameter characteristic of the considered nanotube which can be used for comparative purposes. Moreover, the goodness of the fits between the model and the measured intensity profiles suggests that the assumption of isotropy might be justifiable.

The absolute value of the absorption coefficient may also depend on the details of the image acquisition, such as intensity of the beam, focusing, etc. For comparative purposes, one therefore has to ensure that the images of every series to be compared are obtained under the same conditions.

\subsection{Influence of annealing}

Two series of 15 images taken from a pristine and an annealed multi-walled nanotube samples were analyzed. A $\mathrm{K}-\mathrm{S}$ test was performed in order to test the significance level of the observed variations. As shown in Table 1, annealing leads to a $7 \%$ decrease in the wall mean thickness. This decrease resulted from a modification of the outer radius, while no significant change is observed concerning the inner radius, as assessed by the $\mathrm{K}-\mathrm{S}$ test. The most statistically significant evolution is a $48 \%$ increase in the absorption coefficient during annealing. For a given chemical composition, the denser the material the higher the absorption coefficient should be. One can therefore conclude that the tube walls have become more dense during annealing.

It is far beyond our scope to determine what actually occurs during annealing. Analysis of the two series of 
images is however compatible with the following scenario. In the pristine sample the walls of the nanotubes are made of coarsely piled up graphene sheets. Such a structure is not very dense, and the electrons can pass easily through it leading to a relatively low value of Lambert absorption coefficient $k$. During annealing this structure is rearranged into a more compact or differently organized one [11], with which the electrons interact differently, leading to a higher absorption coefficient.

The rearrangement of the wall occurs together with a decrease in the outer radius, while the inner radius is left unchanged. If one assumes that the absorption coefficient is proportional to the wall density, the quantity $k\left(R_{\mathrm{O}}^{2}-R_{\mathrm{I}}^{2}\right)$ should be proportional to tube weight per unit length. The variation of this quantity during annealing are found not to be statistically significant, as shown in Table 1 . This suggests that the mass per unit length of the tube does not change significantly during annealing, which implies that the tubes neither lengthen nor shorten during the treatment.

\section{Conclusion}

An image analysis methodology has been developed to characterize multi-walled carbon nanotubes from transmission electron microscopy images. The method is divided into three steps. The first step aims at separating the nanotubes from the background. The second step permits isolating their measurable sections, and removing the parts corresponding to their entanglement. The third step involves measurement of the nanotubes. It is performed on the gray level image itself. The intensity of the image across a tube section is determined and a model based on Lambert's law is fitted to it by least squares. This allows two geometrical and one physical characteristics to be determined for each tube, namely, its outer and inner radius and a linear electron absorption coefficient.

The parameters of the preprocessing step have to be chosen by the user, according to the particular type of image. Once this set of parameters has been optimized, the nanotubes to be measured are selected with precisely defined criteria. The total number of images to be used depends mainly on the sampling.

The method has been illustrated by analyzing a multiwalled carbon nanotube sample before and after annealing. It was shown that annealing leads to a lowering of the tube thickness resulting in a significant increase in the electron absorption coefficient. If one assumes that the linear absorption coefficient is proportional to the density, one can further conclude that no significant change of tube length occurs during annealing. However, this conclusion no longer holds if the increase in the linear absorption coefficient results from a more structured arrangement of the wall material.

This method has proven to be successful in characterizing the effect of annealing on multi-walled carbon nanotubes. An electron absorption coefficient can be estimated, which is totally unattainable through visual inspection of micrographs.

\section{Acknowledgements}

C.G. is grateful to the FNRS (National Fund for Scientific Research, Belgium) for a Ph.D. Research Fellow position. This work was supported by the Commission of the European Communities HPRN-CT-2000-00037 NANOCOMP contract, by the Communauté Française de Belgique 00/05-265 Action de Recherche Concertée, and by the Région Wallonne No. 0014622 grant. The authors acknowledge the CRMD Laboratory of Orléans for providing the nanotubes, Professor N. Dupont-Pavlovsky for fruitful discussions and the unknown referee for very thorough and constructive review of the manuscript.

\section{References}

[1] Ijima S. Helical microtubules of graphitic carbon. Nature 1991;354:56-8.

[2] Ijima S. Carbon nanotubes: past, present, and future. Physica B 2002;323:1-5.

[3] Journet C, Bernier P. Production of carbon nanotubes. Appl Phys A 1998;67:1-9.

[4] Soille P. Morphological image analysis: principles and applications. Berlin: Springer; 1999.

[5] Russ J. The image processing handbook. CRC Press; 1999.

[6] Delpeux S, Szotack K, Frakowiak E, Bonnamy S, Béguin F. High yield of pure multiwalled carbon nanotubes from the catalytic decomposition of acetylene on in situ formed cobalt particles. J Nanosci Nanotechnol 2002;2:481-4.

[7] Otsu N. A threshold selection method from gray-level histograms. IEEE Trans Syst Man Cybernet 1979;9(1):62-6.

[8] Adamson AW. A textbook of physical chemistry. New York, San Francisco, London: Academic Press; 1979.

[9] Bougrine A, Dupont-Pavlovsky N, Naji A, Ghanbaja J, Marêché JF, Billaud D. Influence of high temperature treatments on single-walled carbon nanotube structure, morphology and surface properties. Carbon 2001;39:685-95.

[10] Press WH, Brian BP, Teukolsky SA, Vetterling WT. Numerical recipes: the art of scientific computing. Cambridge University Press; 1992.

[11] Rouzaud JN, Oberlin A, Bény-Bassez C. Carbon films: structure and microtexture (optical and electron microscopy, Raman spectroscopy). Thin Solid Films 1983;105(1):75-96. 